\title{
ASYMPTOTICALLY OPTIMAL BOOLEAN FUNCTIONS
}

\author{
KAI-UWE SCHMIDT
}

\begin{abstract}
The largest Hamming distance between a Boolean function in $n$ variables and the set of all affine Boolean functions in $n$ variables is known as the covering radius $\rho_{n}$ of the $\left[2^{n}, n+1\right]$ Reed-Muller code. This number determines how well Boolean functions can be approximated by linear Boolean functions. We prove that

$$
\lim _{n \rightarrow \infty} 2^{n / 2}-\rho_{n} / 2^{n / 2-1}=1,
$$

which resolves a conjecture due to Patterson and Wiedemann from 1983.
\end{abstract}

\section{InTRODUCTION AND RESUlts}

The Hamming distance of two Boolean functions $F, G: \mathbb{F}_{2}^{n} \rightarrow \mathbb{F}_{2}$ is

$$
d(F, G)=\#\left\{y \in \mathbb{F}_{2}^{n}: F(y) \neq G(y)\right\} .
$$

Put

$$
\rho_{n}=\max _{F} \min _{G} d(F, G)
$$

where the maximum is over all functions $F$ from $\mathbb{F}_{2}^{n}$ to $\mathbb{F}_{2}$ and the minimum is over all $2^{n+1}$ affine functions $G$ from $\mathbb{F}_{2}^{n}$ to $\mathbb{F}_{2}$. Then $\rho_{n}$ equals the covering radius of the $\left[2^{n}, n+1\right]$ Reed-Muller code, whose determination is one of the oldest and most difficult open problems in coding theory [6], [14, [17]. We refer to [4] for background on the covering radius of codes in general and its combinatorial and geometric significance. The determination of $\rho_{n}$ also answers the question of how well Boolean functions can be approximated by linear functions, which is of significance in cryptography [3]. One can also interprete $\rho_{n}$ in terms of the Fourier coefficients of Boolean functions (see Section 2).

It is convenient to define

$$
\mu_{n}=2^{n / 2}-\rho_{n} / 2^{n / 2-1} .
$$

An averaging argument shows that $\mu_{n} \geq 1$ (see Section 21) and a simple recursive construction involving functions of the form $F(y)+u v$ on $\mathbb{F}_{2}^{n+2}$ shows that $\mu_{n+2} \leq \mu_{n}$. The fact that $\mu_{2}=1$ implies that $\mu_{n}=1$ for all even $n$; the functions attaining the minimum are known as bent functions and these have been studied extensively for more than forty years [15], [12].

Date: 22 November 2017.

2010 Mathematics Subject Classification. Primary: 11T71; Secondary: 94B05, 06E30.

K.-U. Schmidt is partly supported by German Research Foundation (DFG). 
We are interested in the case that $n$ is odd. Since $\mu_{1}=\sqrt{2}$, we have $1 \leq \mu_{n} \leq \sqrt{2}$. It is known that equality holds in the upper bound for $n=3$ (trivial), for $n=5$ [1], and for $n=7$ [13], 7]. It was suggested in [6] that $\mu_{n}=\sqrt{2}$ for all odd $n$, which was disproved by Patterson and Wiedemann [14, by showing that

$$
\mu_{n} \leq \sqrt{729 / 512}=1.19 \ldots \text { for each } n \geq 15 .
$$

More recently it was shown by Kavut and Yücel [8] that

$$
\mu_{n} \leq \sqrt{49 / 32}=1.23 \ldots \text { for each } n \geq 9 .
$$

Patterson and Wiedemann [14] also conjectured that $\lim _{n \rightarrow \infty} \mu_{n}=1$. However no improvement of (1) for large $n$ has been found since this conjecture has been posed in 1983. We shall prove that this conjecture is true.

Theorem 1. We have $\lim _{n \rightarrow \infty} \mu_{n}=1$.

Several researchers (for example in [16], 5], [11]) also investigated

$$
\rho_{n}^{\prime}=\max _{F} \min _{G} d(F, G)
$$

where now the maximum is over all balanced functions $F$ from $\mathbb{F}_{2}^{n}$ to $\mathbb{F}_{2}$ (which means that $F$ takes the values 0 and 1 equally often) and the minimum is still over all affine functions $G$ from $\mathbb{F}_{2}^{n}$ to $\mathbb{F}_{2}$. Put

$$
\mu_{n}^{\prime}=2^{n / 2}-\rho_{n}^{\prime} / 2^{n / 2-1} .
$$

Slight modifications of our proof of Theorem 1 lead to the following result, which proves a conjecture due to Dobbertin [5, Conjecture B] from 1995.

Theorem 2. We have $\lim _{n \rightarrow \infty} \mu_{n}^{\prime}=1$.

\section{Proof of MAin Result}

In what follows, we identify $\mathbb{F}_{2}^{n}$ with $\mathbb{F}_{2^{n}}$ and consider functions $f: \mathbb{F}_{2^{n}} \rightarrow$ $\mathbb{C}$. Let $\psi: \mathbb{F}_{2^{n}} \rightarrow \mathbb{C}$ be the canonical additive character of $\mathbb{F}_{2^{n}}$, which is given by $\psi(y)=(-1)^{\operatorname{Tr}(y)}$, where $\operatorname{Tr}$ is the absolute trace function on $\mathbb{F}_{2^{n}}$. The Fourier transform of $f$ is the function $\hat{f}: \mathbb{F}_{2^{n}} \rightarrow \mathbb{C}$ given by

$$
\hat{f}(a)=\frac{1}{2^{n / 2}} \sum_{y \in \mathbb{F}_{2^{n}}} f(y) \psi(a y) .
$$

It is well known [3] and readily verified that

$$
\mu_{n}=\min _{f} \max _{a \in \mathbb{F}_{2^{n}}}|\hat{f}(a)|,
$$

where the minimum is over all functions $f: \mathbb{F}_{2^{n}} \rightarrow\{-1,1\}$. From Parseval's identity

$$
\sum_{a \in \mathbb{F}_{2^{n}}}|\hat{f}(a)|^{2}=\sum_{y \in \mathbb{F}_{2^{n}}}|f(y)|^{2}
$$

it follows now that $\mu_{n} \geq 1$. 
We shall construct functions $f$ with image $\{-1,1\}$ for which $|\hat{f}(a)|$ is small for all $a \in \mathbb{F}_{2^{n}}$. Let $H$ be a (multiplicative) subgroup of $\mathbb{F}_{2^{n}}^{*}$ of index $v$ and define the indicator function of $H$ on $\mathbb{F}_{2^{n}}$ by

$$
\mathbb{1}_{H}(y)= \begin{cases}1 & \text { for } y \in H \\ 0 & \text { otherwise. }\end{cases}
$$

Let $h: H \rightarrow\{-1,1\}$ be a function to be specified later. Let $T$ be a complete system of coset representatives of $H$ in $\mathbb{F}_{2^{n}}^{*}$ and let $g: T \rightarrow\{0,-1,1\}$ be a function satisfying $g(z)=0$ if and only if $z \in H$ and such that $g$ is balanced, which means that the images -1 and 1 occur equally often. We define $f: \mathbb{F}_{2^{n}} \rightarrow\{-1,1\}$ by $f(0)=1$ and

$$
f(y)=\mathbb{1}_{H}(y) h(y)+\sum_{z \in T} \mathbb{1}_{H}(y / z) g(z) \quad \text { for } y \in \mathbb{F}_{2^{n}}^{*} .
$$

Note that $f$ is constant on the cosets of $H$, except for $H$ itself. Such functions were also used by Patterson and Wiedemann [14] in their proof of (1) and have been also studied in several other papers, for example in [2.

Recall that $\operatorname{ord}_{v}(a)$ for integers $v$ and $a$ with $v>0$ and $\operatorname{gcd}(a, v)=1$ is the smallest positive integer $t$ such that $a^{t} \equiv 1(\bmod v)$.

Proposition 3. Let e be a positive integer and let $v=7^{e}$. Then there exists an odd multiple $n$ of $\operatorname{ord}_{v}(2)$ and a function $h: H \rightarrow\{-1,1\}$ such that the function $f: \mathbb{F}_{2^{n}} \rightarrow\{-1,1\}$, defined above, satisfies

$$
\max _{a \in \mathbb{F}_{2^{n}}}|\hat{f}(a)| \leq 1+12 \sqrt{\frac{\log (2 v)}{v}} .
$$

A routine induction shows that $\operatorname{ord}_{7^{e}}(2)$ equals $\phi\left(7^{e}\right) / 2=3 \cdot 7^{e-1}$, and so is odd, for all positive integers $e$ (where $\phi$ is the Euler totient function). Now let $e$ tend to infinity in Proposition 3 and use $\mu_{n}=1$ for all even $n$ and the inequality $1 \leq \mu_{n+2} \leq \mu_{n}$ for all $n$ to deduce Theorem 1 from Proposition 3 ,

Remark. Proposition 3 remains true if 7 is replaced by an arbitrary prime $q \equiv 3(\bmod 4)$ such that $\operatorname{ord}_{q^{e}}(2)=\phi\left(q^{e}\right) / 2$ for each $e \in\{1,2\}$ (which ensures that this identity holds for all positive integers $e$ ). The first primes of this form are $7,23,47,71,79$, but it is not known whether there are infinitely many such primes. We choose $q=7$ to keep our proof simple.

To prove Proposition [3, we define functions $f_{1}, f_{2}: \mathbb{F}_{2^{n}} \rightarrow\{0,-1,1\}$ by

$$
\begin{aligned}
& f_{1}(y)=\mathbb{1}_{H}(y) h(y), \\
& f_{2}(y)=\sum_{z \in T} \mathbb{1}_{H}(y / z) g(z),
\end{aligned}
$$

so that $f(y)=f_{1}(y)+f_{2}(y)$ for all $y \in \mathbb{F}_{2^{n}}^{*}$ and $\hat{f}(a)=2^{-n / 2}+\hat{f}_{1}(a)+\hat{f}_{2}(a)$ for all $a \in \mathbb{F}_{2^{n}}$. We shall see that bounding $\left|\hat{f}_{1}(a)\right|$ is not difficult using known results from probabilistic combinatorics. Bounding $\left|\hat{f}_{2}(a)\right|$ requires a little more work. 
For a multiplicative character $\chi$ of $\mathbb{F}_{2^{n}}$, the Gauss sum $G(\chi)$ is defined to be

$$
G(\chi)=\sum_{y \in \mathbb{F}_{2}^{*}} \psi(y) \chi(y) .
$$

It is well known that $|G(\chi)|=2^{n / 2}$ if $\chi$ is nontrivial (which means that $\chi(y) \neq 1$ for some $\left.y \in \mathbb{F}_{2^{n}}^{*}\right)[10$, Theorem 5.11].

We begin with the following elementary lemma.

Lemma 4. Let $\epsilon>0$ and suppose that, for all nontrivial multiplicative characters $\chi$ of $\mathbb{F}_{2^{n}}$ of order dividing $v$, we have

$$
\left|\frac{G(\chi)}{2^{n / 2}}-1\right| \leq \epsilon
$$

Then we have

$$
\max _{a \in \mathbb{F}_{2^{n}}}\left|\hat{f}_{2}(a)\right| \leq 1+\epsilon v
$$

Proof. Since $g$ is balanced, we have $\hat{f}_{2}(0)=0$, so let $a \in \mathbb{F}_{2^{n}}^{*}$. Let $\chi$ be a multiplicative character of $\mathbb{F}_{2^{n}}$ of order $v$. Then the indicator function $\mathbb{1}_{H}$ satisfies

$$
\mathbb{1}_{H}(y)=\frac{1}{v} \sum_{j=0}^{v-1} \chi^{j}(y) \quad \text { for each } y \in \mathbb{F}_{2^{n}}^{*} .
$$

Therefore we have

$$
\begin{aligned}
\sum_{y \in \mathbb{F}_{2^{n}}} \mathbb{1}_{H}(y) \psi(a y) & =\frac{1}{v} \sum_{j=0}^{v-1} \sum_{y \in \mathbb{F}_{2^{*}}^{*}} \psi(a y) \chi^{j}(y) \\
& =\frac{1}{v} \sum_{j=0}^{v-1} \chi^{j}\left(a^{-1}\right) \sum_{y \in \mathbb{F}_{2}^{*}} \psi(y) \chi^{j}(y) \\
& =\frac{1}{v} \sum_{j=0}^{v-1} \bar{\chi}^{j}(a) G\left(\chi^{j}\right),
\end{aligned}
$$

which we use to obtain

$$
\begin{aligned}
2^{n / 2} \hat{f}_{2}(a) & =\sum_{y \in \mathbb{F}_{2^{n}}} \sum_{z \in T} \mathbb{1}_{H}(y / z) g(z) \psi(a y) \\
& =\sum_{z \in T} g(z) \sum_{y \in \mathbb{F}_{2^{n}}} \mathbb{1}_{H}(y) \psi(a y z) \\
& =\frac{1}{v} \sum_{z \in T} g(z) \sum_{j=0}^{v-1} \bar{\chi}^{j}(a z) G\left(\chi^{j}\right) \\
& =\frac{1}{v} \sum_{j=0}^{v-1} G\left(\chi^{j}\right) \bar{\chi}^{j}(a) \sum_{z \in T} g(z) \bar{\chi}^{j}(z) .
\end{aligned}
$$


Now write $G\left(\chi^{j}\right)=2^{n / 2}\left(1+\gamma_{j}\right)$, so that $\left|\gamma_{j}\right| \leq \epsilon$ for all $j \in\{1, \ldots, v-1\}$ by our assumption. Since $G\left(\chi^{0}\right)=-1$, we obtain $\hat{f}_{2}(a)=M(a)+E(a)$, where

$$
\begin{aligned}
M(a) & =\frac{1}{v} \sum_{j=1}^{v-1} \bar{\chi}^{j}(a) \sum_{z \in T} g(z) \bar{\chi}^{j}(z)-\frac{1}{2^{n / 2} v} \sum_{z \in T} g(z) \\
& =\frac{1}{v} \sum_{z \in T} g(z) \sum_{j=1}^{v-1} \bar{\chi}^{j}(a z) \\
& =\frac{1}{v} \sum_{z \in T} g(z) \sum_{j=0}^{v-1} \bar{\chi}^{j}(a z)-\frac{1}{v} \sum_{z \in T} g(z) \\
& =g(b) \quad \text { for } b \in T \text { such that } a b \in H,
\end{aligned}
$$

using that $g$ is balanced and (2) again, and

$$
|E(a)|=\left|\frac{1}{v} \sum_{j=1}^{v-1} \gamma_{j} \bar{\chi}^{j}(a) \sum_{z \in T} g(z) \bar{\chi}^{j}(z)\right| \leq \epsilon v .
$$

This gives the required result.

The following explicit evaluation of certain Gauss sums [9, Proposition 4.2] (see also [20, Theorem 4.1]) will help us to control the error term in Lemma4,

Lemma 5 ([9, Proposition 4.2]). Let $q>3$ be a prime satisfying $q \equiv 3$ $(\bmod 4)$. Let $d$ be a positive integer, write $k=\phi\left(q^{d}\right) / 2$, and let $p$ be a prime such that $\operatorname{ord}_{q^{d}}(p)=k$. Let $\tau$ be a multiplicative character of $\mathbb{F}_{p^{k}}$ of order $q^{d}$ and let $h$ be the class number of $\mathbb{Q}(\sqrt{-q})$. Then

$$
G(\tau)=\frac{1}{2}(a+b \sqrt{-q}) p^{(k-h) / 2},
$$

where $a$ and $b$ are integers satisfying $a, b \not \equiv 0(\bmod p), a^{2}+b^{2} q=4 p^{h}$, and $a p^{(k-h) / 2} \equiv-2(\bmod q)$.

We shall apply Lemma 5 with $p=2$ and $q=7$. Since the class number of $\mathbb{Q}(\sqrt{-7})$ equals 1 and

$$
2^{\left(\phi\left(7^{d}\right) / 2-1\right) / 2} \equiv 2 \quad(\bmod 7)
$$

for all positive integers $d$, we find that $a=-1$ and $b^{2}=1$ in this case. As noted after Proposition 3, we have $\operatorname{ord}_{7^{d}}(2)=\phi\left(7^{d}\right) / 2$ for all positive integers $d$, so that the hypothesis in Lemma 5 is satisfied for $p=2$ and $q=7$.

As a corollary to Lemma 5, we obtain the following lemma.

Lemma 6. Let $e$ and $d$ be integers satisfying $1 \leq d \leq e$ and write $m=$ $\operatorname{ord}_{7}(2)$. Let $\chi$ be a multiplicative character of $\mathbb{F}_{2^{s m}}$ of order $7^{d}$. Then

$$
\frac{G(\chi)}{2^{s m / 2}}=-(-1)^{s}\left(\frac{-1 \pm \sqrt{-7}}{2^{3 / 2}}\right)^{7^{e-d} s},
$$

where the sign depends on $\chi$. 
Proof. Write $k=\operatorname{ord}_{7^{d}}(2)$ and let $\tau$ be the multiplicative character of $\mathbb{F}_{2^{k}}$ such that $\chi$ is the lifted character of $\tau$, by which we mean that $\chi=\tau \circ \mathrm{N}$, where $\mathrm{N}$ is the field norm from $\mathbb{F}_{2^{s m}}$ to $\mathbb{F}_{2^{k}}$. Lemma 5 and the preceding discussion implies that

$$
G(\tau)=2^{(k-3) / 2}(-1 \pm \sqrt{-7}) .
$$

From the Davenport-Hasse theorem [10, Theorem 5.14] we find that

$$
G(\chi)=-(-1)^{s m / k}\left[2^{(k-3) / 2}(-1 \pm \sqrt{-7})\right]^{s m / k},
$$

and the lemma follows since $m / k=\phi\left(7^{e}\right) / \phi\left(7^{d}\right)=7^{e-d}$.

The next lemma gives the desired control for the error term in Lemma 4 .

Lemma 7. Let e be a positive integer, let $v=7^{e}$, and write $m=\operatorname{ord}_{v}(2)$. Let $\epsilon>0$. Then there is an infinite set $S$ of odd positive integers such that, for all $s \in S$ and all nontrivial multiplicative characters $\chi$ of $\mathbb{F}_{2^{\text {sm }}}$ of order dividing $v$, we have

$$
|\arg G(\chi)| \leq \epsilon
$$

Here, $\arg (\xi) \in(-\pi, \pi]$ is the principal angle of a nonzero complex number $\xi$.

Proof. Let $\tau$ be a multiplicative character of $\mathbb{F}_{2^{m}}$ of order $v$. Since the units of the ring of algebraic integers in $\mathbb{Q}(\sqrt{-7})$ are \pm 1 , we find from Lemma 6 that $G(\tau) / 2^{m / 2}$ is not a root of unity. Therefore Weyl's uniform distribution theorem [19, Satz 2] implies that $\left(\left[G(\tau) / 2^{m / 2}\right]^{2 i}\right)_{i \in \mathbb{N}}$, and therefore also $\left(\left[G(\tau) / 2^{m / 2}\right]^{2 i+1}\right)_{i \in \mathbb{N}}$, is uniformly distributed on the complex unit circle. Hence there is an infinite set $S$ of odd positive integers such that

$$
\left|\arg \left(G(\tau)^{s}\right)\right| \leq \frac{\epsilon}{7^{e-1}}
$$

for all $s \in S$.

Let $s \in S$ and let $\tau^{\prime}$ be the lifted character of $\tau$ to $\mathbb{F}_{2^{s m}}$, namely $\tau^{\prime}=\tau \circ \mathrm{N}$, where $\mathrm{N}$ is the field norm from $\mathbb{F}_{2^{s m}}$ to $\mathbb{F}_{2^{m}}$. Then $\tau^{\prime}$ has order $v=7^{e}$ and the Davenport-Hasse theorem [10, Theorem 5.14] states $G\left(\tau^{\prime}\right)=G(\tau)^{s}$, so that

$$
\left|\arg G\left(\tau^{\prime}\right)\right| \leq \frac{\epsilon}{7^{e-1}}
$$

Now let $\chi$ be a multiplicative character of $\mathbb{F}_{2^{s m}}$ of order $7^{d}$, where $1 \leq d \leq e$. Then by Lemma 6 we have

$$
|\arg G(\chi)| \leq 7^{e-d}\left|\arg G\left(\tau^{\prime}\right)\right|,
$$

which completes the proof.

We need one more classical result from probabilistic combinatorics due to Spencer [18]. 
Lemma 8 ([18, Theorem 7]). Let $A$ be a matrix of size $M \times N$ satisfying $M \geq N$ with real-valued entries of absolute value at most 1 . Then, for all sufficiently large $N$, there exists $u \in\{-1,1\}^{N}$ such that

$$
\|A u\| \leq 11 \sqrt{N \log (2 M / N)}
$$

where $\|\cdot\|$ is the maximum norm on $\mathbb{R}^{M}$.

We now prove Proposition 3.

Proof of Proposition [3. Write $m=\operatorname{ord}_{v}(2)$. Lemma 7 implies that, for all $\epsilon>0$, there is an infinite set $S$ of odd positive integers such that

$$
\left|\frac{G(\chi)}{2^{s m / 2}}-1\right| \leq \epsilon
$$

for all $s \in S$ and all nontrivial multiplicative characters $\chi$ of $\mathbb{F}_{2^{s m}}$ of order dividing $v$. Writing $n=s m$ and taking $\epsilon=\frac{1}{2} \sqrt{\log (2 v) / v^{3}}$, Lemma 4 then implies that

$$
\max _{a \in \mathbb{F}_{2^{n}}}\left|\hat{f}_{2}(a)\right| \leq 1+\frac{1}{2} \sqrt{\frac{\log (2 v)}{v}}
$$

for infinitely many odd positive integers $n$.

It remains to consider $\hat{f}_{1}$. Since

$$
\hat{f}_{1}(a)=\frac{1}{2^{n / 2}} \sum_{y \in H} h(y) \psi(a y),
$$

we find from Lemma 8 with $M=2^{n}$ and $N=\left(2^{n}-1\right) / v$ that, for all sufficiently large $n$, there exists $h: H \rightarrow\{-1,1\}$ such that

$$
\max _{a \in \mathbb{F}_{2^{n}}}\left|\hat{f}_{1}(a)\right| \leq 11 \sqrt{\frac{\log (2 v)}{v}} .
$$

Since $\hat{f}(a)=2^{-n / 2}+\hat{f}_{1}(a)+\hat{f}_{2}(a)$ for all $a \in \mathbb{F}_{2^{n}}$, there is an odd integer $n$ that is a multiple of $m=\operatorname{ord}_{v}(2)$ such that

$$
\max _{a \in \mathbb{F}_{2^{n}}}|\hat{f}(a)| \leq 1+12 \sqrt{\frac{\log (2 v)}{v}},
$$

as required.

We now comment on the required modifications of our proof to prove Theorem 2. The function $h$ identified in the proof of Proposition 3 satisfies

$$
\left|\sum_{y \in H} h(y)\right| \leq 11 \sqrt{2^{n} \frac{\log (2 v)}{v}} .
$$

Therefore we have to change at most $6 \sqrt{2^{n} \log (2 v) / v}$ values of the function $h$ to get

$$
\sum_{y \in H} h(y)=-1
$$


This increases $\left|\hat{f}_{1}(a)\right|$ by at most $12 \sqrt{\log (2 v) / v}$. The resulting function $f$ is balanced and satisfies

$$
\max _{a \in \mathbb{F}_{2^{n}}}|\hat{f}(a)| \leq 1+24 \sqrt{\frac{\log (2 v)}{v}} .
$$

Using $1 \leq \mu_{n+2}^{\prime} \leq \mu_{n}^{\prime}$, this shows that $\lim _{i \rightarrow \infty} \mu_{2 i+1}^{\prime}=1$. We combine this with $\lim _{i \rightarrow \infty} \mu_{2 i}^{\prime}=1$, which was already shown in [5], but also follows from our argument using further slight modifications, to obtain a proof of Theorem 2 .

\section{ACKNOWLEDGEMENTS}

I would like to thank James A. Davis for many valuable discussions in the early stage of this research.

\section{REFERENCES}

[1] E. R. Berlekamp and L. R. Welch. Weight distributions of the cosets of the $(32,6)$ Reed-Muller code. IEEE Trans. Inform. Theory, IT-18(1):203-207, 1972.

[2] J. Bringer, V. Gillot, and P. Langevin. Exponential sums and Boolean functions. In Proceedings of Boolean Functions: Cryptography and Applications, pages 177-185, Rouen, 2005.

[3] C. Carlet. Boolean functions for cryptography and error-correcting codes. In Y. Crama and P. L. Hammer, editors, Boolean models and methods in mathematics, computer science, and engineering, pages 257-397. 2010.

[4] G. Cohen, I. Honkala, S. Litsyn, and A. Lobstein. Covering codes, volume 54 of NorthHolland Mathematical Library. North-Holland Publishing Co., Amsterdam, 1997.

[5] H. Dobbertin. Construction of bent functions and balanced Boolean functions with high nonlinearity. In B. Preneel, editor, Fast Software Encryption: Second International Workshop Leuven, Belgium, December 14-16, 1994 Proceedings, pages 61-74. Springer Berlin Heidelberg, 1995.

[6] T. Helleseth, T. Kløve, and J. Mykkeltveit. On the covering radius of binary codes. IEEE Trans. Inform. Theory, 24(5):627-628, 1978.

[7] X.-D. Hou. Covering radius of the Reed-Muller code $R(1,7)$ - a simpler proof. $J$. Combin. Theory Ser. A, 74(2):337-341, 1996.

[8] S. Kavut and M. D. Yücel. 9-variable Boolean functions with nonlinearity 242 in the generalized rotation symmetric class. Inform. and Comput., 208(4):341-350, 2010.

[9] P. Langevin. Calculs de certaines sommes de Gauss. J. Number Theory, 63(1):59-64, 1997.

[10] R. Lidl and H. Niederreiter. Finite fields, volume 20 of Encyclopedia of Mathematics and its Applications. Cambridge University Press, Cambridge, 2nd edition, 1997.

[11] S. Maitra and P. Sarkar. Modifications of Patterson-Wiedemann functions for cryptographic applications. IEEE Trans. Inform. Theory, 48(1):278-284, 2002.

[12] S. Mesnager. Bent functions: Fundamentals and results. Springer, 2016.

[13] J. J. Mykkeltveit. The covering radius of the $(128,8)$ Reed-Muller code is 56. IEEE Trans. Inform. Theory, 26(3):359-362, 1980.

[14] N. J. Patterson and D. H. Wiedemann. The covering radius of the $\left(2^{15}, 16\right)$ ReedMuller code is at least 16276 . IEEE Trans. Inform. Theory, 29(3):354-356, 1983. Corrected in: IEEE Trans. Inform. Theory, 36(2):443, 1990.

[15] O. S. Rothaus. On "bent" functions. J. Combin. Theory Ser. A, 20(3), 1976.

[16] J. Seberry, Z. M. Zhang, and Y. Zheng. Nonlinearity and propagation characteristics of balanced Boolean functions. Inform. and Comput., 119(1):1-13, 1995. 
[17] N. Sloane. Unsolved problems related to the covering radius of codes. In T. Cover and B. Gopinath, editors, Open Problems in Communication and Computation, pages 51-56. Springer New York, 1987.

[18] J. Spencer. Six standard deviations suffice. Trans. Amer. Math. Soc., 289(2):679-706, 1985.

[19] H. Weyl. Über die Gleichverteilung von Zahlen mod. Eins. Math. Ann., 77(3):313-352, 1916.

[20] J. Yang and L. Xia. Complete solving of explicit evaluation of Gauss sums in the index 2 case. Sci. China Math., 53(9):2525-2542, 2010.

Department of Mathematics, Paderborn University, Warburger Str. 100, 33098 Paderborn, Germany.

E-mail address, K.-U. Schmidt: kus@math.upb.de 